\title{
ORTHONORMAL SETS WITH NON-NEGATIVE DIRICHLET KERNELS
}

\author{
BY \\ J. J. PRICE
}

1. Introduction. With any orthonormal system $\left\{f_{n}(s)\right\}_{n=0}^{\infty}$ of real-valued functions on a measure space $S$ are associated the Dirichlet kernels

$$
D_{n}(s, t)=\sum_{j=0}^{n-1} f_{j}(s) f_{j}(t), \quad n \geqq 1 .
$$

If these kernels happen to be non-negative, the Lebesgue functions of the system, i.e. the functions

$$
L_{n}(s)=\int_{S}\left|D_{n}(s, t)\right| d t, \quad n \geqq 1,
$$

are much more accessible than otherwise. In particular, if $S=[0,1]$ and $f_{0}(s) \equiv 1$, it is immediate that $L_{n}(s) \equiv 1$ for all $n$. This is exactly the situation one finds in the case of the classical Haar functions (see [1] or [3]). The uniform boundedness of the functions $L_{n}(s)$ leads to a proof that the expansion of a continuous function $g(x)$ on $[0,1]$ in terms of Haar functions converges uniformly to $g(x)$.

It seems natural to ask whether there are other orthonormal sets with the property that $D_{n}(s, t) \geqq 0$ for all $n$. We shall prove that the answer to this question is essentially negative. It will be shown that only the Haar functions and certain minor modifications of them possess non-negative Dirichlet kernels.

2. Definitions. It will be assumed throughout, except in $\$ 5$, that $\mu$ is a totally finite measure $\left.{ }^{1}\right)$ on a space $S$ normalized so that $\mu(S)=1$.

Using the terminology of [2], a partition of $S$ is a finite set $P$ of disjoint subsets of $S$ whose union is $S$. Given two partitions $P_{1}$ and $P_{2}, P_{1} \geqq P_{2}$ if each element of $P_{2}$ is contained in an element of $P_{1}$.

Consider a sequence of partitions of $S$ having the following two properties.

(i) $P_{0} \geqq P_{1} \geqq P_{2} \geqq \cdots$.

(ii) For each $n \geqq 0, P_{n}=\left\{S_{n, 1}, S_{n, 2}, \cdots, S_{n, n+1}\right\}$ where all sets have positive $\mu$-measure and $S_{0,1}=S$.

These conditions amount to assuming that $P_{0}=\{S\}$ and $P_{n+1}$ is obtained from $P_{n}$ by splitting one of the sets $S_{n, j}$, say for $j=k(n)$, into two subsets of

Presented to the Society, April 24, 1959; received by the editors May 4, 1959.

(1) See [2] for measure-theoretic definitions. 
positive measure. The enumeration can always be arranged so that $S_{n, k(n)}$ splits to form $S_{n+1,1}$ and $S_{n+1,2}$. The sets $S_{n, j}(1 \leqq j \leqq n+1, j \neq k(n))$ are relabeled and become the sets $S_{n+1, i}(3 \leqq i \leqq n+2)$. To avoid cumbersome subscripts, we shall use $k$ instead of $k(n)$.

With such a sequence of partitions, we associate an orthonormal set $\left\{f_{n}(s)\right\}_{n=0}^{\infty}$ in $L^{2}(S, \mu)$ as follows. Put $f_{0}(s) \equiv 1$. Let $\mu\left(S_{n, j}\right)=\mu_{n, j}$ and define for $n \geqq 1$

$$
f_{n}(s)=\left\{\begin{array}{cc}
\left(\frac{\mu_{n, 2}}{\mu_{n, 1} \mu_{n-1, k}}\right)^{1 / 2}, & s \in S_{n, 1}, \\
-\left(\frac{\mu_{n, 1}}{\mu_{n, 2} \mu_{n-1, k}}\right)^{1 / 2}, & s \in S_{n, 2}, \\
0, & \text { otherwise. }
\end{array}\right.
$$

A set of functions defined in this way will be called a Haar system on $(S, \mu)$. It is easy to verify that a Haar system is an orthonormal set in $L^{2}(S, \mu)$.

These are obvious generalizations of the orthonormal sets introduced by Haar in [1]. There, $S$ is the unit interval, $\mu$ is Lebesgue measure, and the $S_{n, j}$ are sub-intervals. That set in which $\mu_{n, 1}=\mu_{n, 2}(n \geqq 1)$ is the one usually called "the Haar functions." (Strictly speaking, the above definition may yield only a subset of the Haar functions. In order to insure completeness, some additional assumption is needed. For instance if $\mu$ is nonatomic, one might require that $\lim _{n \rightarrow \infty} \max _{j} \mu_{n, j}=0$.)

3. Main theorem. For the sake of simplicity, we are going to avoid "almost everywhere" statements. We shall tacitly identify two functions which differ on a set of measure zero. In this way, for example, an essentially bounded function will be considered bounded.

THEOREM 1. Let $H=\left\{f_{n}(s)\right\}_{n=0}^{\infty}$ be a real orthonormal set in $L^{2}(S, \mu)$ with $f_{0}(s) \equiv 1$. The Dirichlet kernels associated with $H$ are non-negative if and only if $H$ is a Haar system on $(S, \mu)$.

The following lemma is needed for the proof of this theorem.

Lemma. Let $T$ be a subset of $S$ having measure $\mu_{T}$, and let $c$ be a positive constant. Define $\mathcal{F}$ to be the class of functions $f \in L^{2}(T, \mu)$ satisfying

$$
\int_{T} f d \mu=0
$$

and

$$
f(s) f(t) \geqq-c,
$$$$
(s, t) \in T \times T \text {. }
$$

Then

$$
\int_{T} f^{2} d \mu \leqq c \mu_{T}, \quad f \in \mathcal{F}
$$


The equality in $(\mathrm{C})$ holds for $f^{*} \in \mathcal{F}$ if and only if there are two complementary subsets $T_{1}$ and $T_{2}$ of $T$ with measures $\mu_{1}$ and $\mu_{2}$ such that

$$
f^{*}(s)= \begin{cases}\left(\frac{c \mu_{2}}{\mu_{1}}\right)^{1 / 2}, & s \in T_{1}, \\ -\left(\frac{c \mu_{1}}{\mu_{2}}\right)^{1 / 2}, & s \in T_{2} .\end{cases}
$$

Proof. Given $f \in \mathcal{F}, f=f_{1}-f_{2}$ where $f_{1}(s)=\max \{f(s), 0\}$ and $f_{2}(s)$ $=\max \{-f(s), 0\}$. For $i=1,2$, let $T_{i}=\left\{s \mid f_{i}(s)>0\right\}$ and let $T_{3}=\{s \mid f(s)=0\}$. Then the sets $T_{i}$ are disjoint $(i=1,2,3)$ and $\mu_{1}+\mu_{2}+\mu_{3}=\mu_{T}$ where $\mu_{i}=\mu\left(T_{i}\right)$.

Because of assumption (A),

$$
\int_{T_{1}} f_{1} d \mu=\int_{T_{2}} f_{2} d \mu=I(f)
$$

Because of (A) and (B), $f$ is bounded. In fact, if $M_{i}=\sup _{s \in T} f_{i}(s),(i=1,2)$, then $M_{1} M_{2} \leqq c$.

We first maximize $I(f)$. Since $I(f) \leqq M_{i} \mu_{i},(i=1,2)$,

$$
I(f) \leqq \min \left\{M_{1} \mu_{1}, M_{2} \mu_{2}\right\}=\min \left\{M_{1} \mu_{1}, M_{2}\left(\mu_{T}-\mu_{1}-\mu_{3}\right)\right\} .
$$

The function of $\mu_{1}$ on the right above has a unique maximum which occurs when $M_{1} \mu_{1}=M_{2} \mu_{2}$. One easily obtains

$$
I(f) \leqq \max _{\mu_{1}} \min \left\{M_{1} \mu_{1}, M_{2} \mu_{2}\right\}=\frac{M_{1} M_{2}}{M_{1}+M_{2}}\left(\mu_{T}-\mu_{3}\right) .
$$

Now

$$
\int_{T} f^{2} d \mu=\int_{T_{1}} f_{1}^{2} d \mu+\int_{T_{2}} f_{2}^{2} d \mu \leqq M_{1} \int_{T_{1}} f_{1} d \mu+M_{2} \int_{T_{2}} f_{2} d \mu=\left(M_{1}+M_{2}\right) I(f)
$$

and it follows from (2) that

$$
\int_{T} f^{2} d \mu \leqq M_{1} M_{2}\left(\mu_{T}-\mu_{3}\right) \leqq c \mu_{T}
$$

This establishes assertion (C). Furthermore, both equalities hold in (3) if and only if the conditions

$$
f(s) \equiv\left\{\begin{aligned}
M_{1}, & s \in T_{1}, \\
-M_{2}, & s \in T_{2},
\end{aligned}\right.
$$




$$
\begin{gathered}
M_{1} \mu_{1}=M_{2 \mu_{2}}, \quad M_{1} M_{2}=c, \\
\mu_{3}=0,
\end{gathered}
$$

are satisfied simultaneously. (6) shows that $T_{1}$ and $T_{2}$ must be complementary subsets of $T$. From (5),

$$
\begin{aligned}
& M_{1}=\left(M_{1} M_{2}\right)^{1 / 2}\left(\frac{M_{1}}{M_{2}}\right)^{1 / 2}=\left(\frac{c \mu_{2}}{\mu_{1}}\right)^{1 / 2} ; \\
& M_{2}=\left(\frac{c \mu_{1}}{\mu_{2}}\right)^{1 / 2} .
\end{aligned}
$$

Assertion (D) now follows from (4).

4. Proof of Theorem 1. Let $\left\{f_{n}(s)\right\}_{n=0}^{\infty}$ be a Haar system on $(S, \mu)$. We shall prove by induction that for $n \geqq 0$,

$$
D_{n+1}(s, t)=\left\{\begin{array}{lr}
\frac{1}{\mu_{n, j}}, & (s, t) \in S_{n, j} \times S_{n, j},(1 \leqq j \leqq n+1), \\
0, & \text { otherwise. }
\end{array}\right.
$$

This is true for $n=0$ since $f_{0}(s) \equiv 1, D_{1}(s, t) \equiv 1$, and $\mu_{0,1}=\mu(S)=1$. In general

$$
D_{n+2}(s, t)=D_{n+1}(s, t)+f_{n+1}(s) f_{n+1}(t) \text {. }
$$

By definition $f_{n+1}(s) \equiv 0$ outside $S_{n, k}$. Hence $D_{n+2}(s, t)=D_{n+1}(s, t)$ outside $S_{n, k}^{2}=S_{n, k} \times S_{n, k}$. From the induction hypothesis (7) this means

$$
\text { if }(s, t) \notin S_{n, k}^{2}, D_{n+2}(s, t)=\left\{\begin{array}{cr}
\frac{1}{\mu_{n+1, j}}, & (s, t) \in S_{n+1, j}^{2},(3 \leqq j \leqq n+2), \\
0, & \text { otherwise. }
\end{array}\right.
$$

Now consider $(s, t) \in S_{n, k}^{2}=\left(S_{n+1,1} \cup S_{n+1,2}\right)^{2}$. From the definition (1),

$$
f_{n+1}(s) f_{n+1}(t)=\left\{\begin{array}{lr}
\frac{\mu_{n+1,2}}{\mu_{n+1,1} \mu_{n, k}}, & (s, t) \in S_{n+1,1}^{2}, \\
\frac{\mu_{n+1,1}}{\mu_{n+1,2} \mu_{n, k}}, & (s, t) \in S_{n+1,2}^{2}, \\
-\frac{1}{\mu_{n, k}}, & \text { otherwise. }
\end{array}\right.
$$

But by the induction hypothesis and (8),

$$
D_{n+2}(s, t)=f_{n+1}(s) f_{n+1}(t)+\frac{1}{\mu_{n, k}}
$$

when $(s, t) \in S_{n, k}^{2}$. From (10) and (11), we easily find that 


$$
\text { if }(s, t) \in S_{n, k}^{2}, D_{n+2}(s, t)=\left\{\begin{array}{cc}
\frac{1}{\mu_{n+1,1}}, & (s, t) \in S_{n+1,1}^{2}, \\
\frac{1}{\mu_{n+1,2}}, & (s, t) \in S_{n+1,2}^{2}, \\
0, & \text { otherwise. }
\end{array}\right.
$$

Statements (9) and (12) together are equivalent to equation (7) with $n+1$ replaced by $n+2$, completing the induction.

Suppose now that $H=\left\{f_{n}(s)\right\}_{n=0}^{\infty}$ is an orthonormal set in $L^{2}(S, \mu)$, $f_{0}(s) \equiv 1$, and the associated Dirichlet kernels are non-negative. We shall prove by induction that for each $n \geqq 0$ there is a sequence of partitions $P_{0} \geqq P_{1} \geqq P_{2} \geqq \cdots \geqq P_{n}$ satisfying (i) and (ii), that $f_{0}(s), f_{1}(s), \cdots, f_{n}(s)$ are the associated Haar functions, and that (7) holds.

For $n=0$, these assertions are obvious. Assume they are true for $n$. $D_{n+2}(s, t) \geqq 0$. Therefore, from (8) and the induction hypothesis

$$
f_{n+1}(s) f_{n+1}(t) \geqq-D_{n+1}(s, t)=\left\{\begin{array}{cr}
-\frac{1}{\mu_{n, j}}, & (s, t) \in S_{n, j}^{2},(1 \leqq j \leqq n+1), \\
0, & \text { otherwise. }
\end{array}\right.
$$

We claim that $f_{n+1}(s) \equiv 0$ on all but one of the sets $S_{n, j}(1 \leqq j \leqq n+1)$. Since $f_{n+1}(s) \not \equiv 0$ on $S$, we can find a set $S_{n, k}$ on which $f_{n+1}(s) \not \equiv 0$. Suppose $f_{n+1}(s) \not \equiv 0$ outside $S_{n, k}$. Then there are points $s \in S_{n, k}$ and points $t \notin S_{n, k}$ where the function is nonzero. For any pair $(s, t)$ of such points, we have from (13) that $f_{n+1}(s) f_{n+1}(t)>0$. It follows that either $f_{n+1}(s) \geqq 0$ or $f_{n+1}(s) \leqq 0$ for all $s \in S$. But this is impossible since

$$
\int_{S} f_{n+1} d \mu=\int_{S} f_{0} f_{n+1} d \mu=0 .
$$

Therefore, $f_{n+1}(s) \equiv 0$ outside $S_{n, k}$.

Consider now the restriction of $f_{n+1}$ to the subset $S_{n, k}$. The following three statements hold.

$$
\begin{aligned}
\int_{S_{n, k}} f_{n+1} d \mu & =0 . \\
f_{n+1}(s) f_{n+1}(t) & \geqq-\frac{1}{\mu_{n, k}} . \\
\int_{S_{n, k}} f_{n+1}^{2} d \mu & =1 .
\end{aligned}
$$

(A) and (C) express orthonormality; (B) is a restatement of part of (13). Now (A) and (B) are precisely the conditions of the lemma with $\mu_{T}=\mu_{n, k}$ and $c=1 / \mu_{n, k}$. We conclude 


$$
\int_{S_{n, k}} f_{n+1}^{2} d \mu \leqq c \mu_{T}=1 .
$$

But now (C) shows that $f_{n+1}$ is maximal in the sense of the lemma. Therefore, there exist two complementary subsets $S_{n+1,1}$ and $S_{n+1,2}$ of $S_{n, k}$ having measures $\mu_{n+1,1}$ and $\mu_{n+1,2}$ such that

$$
f_{n+1}(s)=\left\{\begin{array}{cc}
\left(\frac{\mu_{n+1,2}}{\mu_{n+1,1} \mu_{n, k}}\right)^{1 / 2}, & s \in S_{n+1,1}, \\
-\left(\frac{\mu_{n+1,1}}{\mu_{n+1,2} \mu_{n, k}}\right)^{1 / 2}, & s \in S_{n+1,2} \\
0, & \text { otherwise. }
\end{array}\right.
$$

For $3 \leqq j \leqq n+2$, define sets $S_{n+1, j}$ to be the sets $S_{n, i}(1 \leqq i \leqq n+1, i \neq k)$ taken in any order. Clearly $P_{n+1}=\left\{S_{n+1, j}\right\}_{j=1}^{n+2}$ is a partition of $(S, \mu), P_{0} \geqq P_{1} \geqq \cdots$ $\geqq P_{n} \geqq P_{n+1}$ is an extension of the sequence of partitions already defined, $f_{n+1}(s)$ is the Haar function associated with $P_{n+1}$, and (7) follows for $n+2$ exactly as in the first part of the proof. This completes the proof by induction that $H$ is a Haar system on $(S, \mu)$.

5. An extension of Theorem 1 . We now drop the assumption that $(S, \mu)$ is totally finite and weaken the restriction that $f_{0}(s) \equiv 1$.

Theorem 2. Let $\left\{f_{n}(s)\right\}_{n=0}^{\infty}$ be a real orthonormal set in $L^{2}(S, \mu)$. The associated Dirichlet kernels are non-negative if and only if and

(A) $f_{0}(s)$ is either non-negative or nonpositive

(B) for each $n \geqq 0, f_{n}(s)=f_{0}(s) \phi_{n}(s)$ where $\left\{\phi_{n}(s)\right\}_{n=0}^{\infty}$ is a Haar system on $(S, \nu)$ and $d \nu=f_{0}^{2}(s) d \mu$.

Proof. To prove sufficiency of these conditions, note that $(S, \nu)$ is totally finite and $\nu(S)=1$. Therefore, by Theorem 1 ,

$$
\sum_{j=0}^{n-1} \phi_{j}(s) \phi_{j}(t) \geqq 0 .
$$

But

$$
D_{n}(s, t)=\sum_{j=0}^{n-1} f_{j}(s) f_{j}(t)=f_{0}(s) f_{0}(t) \sum_{j=0}^{n-1} \phi_{j}(s) \phi_{j}(t)
$$

Since $f_{0}(s) f_{0}(t) \geqq 0$ from condition (A), $D_{n}(s, t) \geqq 0$.

Next, we show necessity. Since $f_{0}(s) f_{0}(t)=D_{1}(s, t) \geqq 0$ condition (A) holds.

Let $Z=\left\{s \mid f_{0}(s)=0\right\}$. We shall prove by induction that $f_{n}(s) \equiv 0$ on $Z$ for $n \geqq 0$. Suppose this is true for $0,1,2, \cdots, n$. 


$$
0 \leqq D_{n+2}(s, t)=f_{n+1}(s) f_{n+1}(t)+\sum_{j=0}^{n} f_{j}(s) f_{j}(t) .
$$

If $s \in Z$, the second term on the right vanishes because of the induction hypothesis, so that $f_{n+1}(s) f_{n+1}(t) \geqq 0$. Suppose $f_{n+1}(s) \neq 0$ on $Z$. Let $s \in Z$ and $t \in S$ be points where the function is nonzero. Then $f_{n+1}(s) f_{n+1}(t)>0$. It follows that either $f_{n+1}(s) \geqq 0$ or $f_{n+1}(s) \leqq 0$ for all $s \in S$. But this is impossible because then either $f_{0}(s) f_{n+1}(s) \geqq 0$ or $f_{0}(s) f_{n+1}(s) \leqq 0$ for all $s$ contradicting the fact that

$$
\int_{S} f_{0} f_{n+1} d \mu=0
$$

Therefore $f_{n+1}(s) \equiv 0$ on $Z$.

We may now define

$$
\phi_{n}(s)=\left\{\begin{array}{c}
\frac{f_{n}(s)}{f_{0}(s)}, \\
0,
\end{array}\right.
$$

Let $d \nu=f_{0}^{2}(s) d \mu$. Then the set $\left\{\phi_{n}(s)\right\}_{n=0}^{\infty}$ is an orthonormal set in $L^{2}(S, \nu)$ for

$$
\int_{S} \phi_{j} \phi_{k} d \nu=\int_{S-Z} \frac{f_{j} f_{k}}{f_{0}^{2}} f_{0}^{2} d \mu=\int_{S} f_{j} f_{k} d \mu=\delta_{j k}
$$

and

$$
\int_{S} \phi_{j}^{2} d \nu=\int_{S-Z}\left(\frac{f_{j}}{f_{0}}\right)^{2} f_{0}^{2} d \mu=\int_{S} f_{j}^{2} d \mu=1
$$

Furthermore, the Dirichlet kernels associated with this set are non-negative since

$$
\sum_{j=0}^{n-1} \phi_{j}(s) \phi_{j}(t)=\left\{\begin{array}{cr}
0, & s \in Z \text { or } t \in Z, \\
\frac{1}{f_{0}(s) f_{0}(t)} \sum_{j=1}^{n-1} f_{j}(s) f_{j}(t)=\frac{D_{n}(s, t)}{D_{1}(s, t)} \geqq 0, & \text { otherwise. }
\end{array}\right.
$$

By Theorem 1, $\left\{\phi_{n}(s)\right\}_{n=0}^{\infty}$ is a Haar system on $(S, \nu)$. Since $f_{n}(s)=f_{0}(s) \phi_{n}(s)$ for $n \geqq 0$, the theorem is proved.

\section{REFERENCES}

1. A. Haar, Zur Theorie der orthogonalen Funktionensysteme, Math. Ann. vol. 69 (1910) pp. 331-371.

2. P. R. Halmos, Measure theory, New York, 1950.

3. S. Kaczmarz and H. Steinhaus, Theorie der Orthogonalreihen, Warsaw, 1935.

Cornell University,

ITHACA, NEW YORK 